\title{
Interactive Guidance by Image Overlay in Robot Assisted Coronary Artery Bypass
}

\author{
Fabien Mourgues ${ }^{1}$, Thierry Vieville ${ }^{1}$, Volkmar Falk ${ }^{2}$, and Ève Coste-Manière ${ }^{1}$ \\ 1 CHIR Team, \\ INRIA, 06902 Sophia-Antipolis, France \\ \{fabien.mourgues, thierry.vieville, eve.coste-maniere\}@inria.fr \\ www.inria.fr/chir \\ 2 HerzZentrum Leipzig GmbH, Germany
}

\begin{abstract}
We present an original visual guidance system in the especially difficult context of robot assisted coronary artery bypass graft. The overlay of a preoperative coronary tree model on the endoscopic images is initialized to help the surgeon to locate himself. Then the surgeon points some landmarks observed in the operating field during the motion of the endoscope. The overlay is corrected in real-time and the landmarks are automatically identified through a multi-model estimation method until the precision necessary to the localization of the surgical targets is reached. The first in vivo experiment, presented here, has shown the efficiency of the system by identifying the main arteries in a few clicks.
\end{abstract}

\section{Introduction}

Minimally invasive surgery takes now advantages of Robot assistance to reduce the trauma and increase the surgeon's gesture precision. The preoperative planning [1] facilitates the approach but several limitations must still be overcome. The tactile feedback is lost and the visual feedback suffers from the keyhole vision provided by the endoscope with confined displacements. Therefore the localization and the identification of the anatomical structures is a difficult task and to provide to the surgeon a guidance system is a challenging research theme.

In Coronary Artery Bypass Graft (CABG), the identification of the vessels is not easy because of the fat partly hiding the arteries, the confined vision and the magnification [2]. We analyzed this Target Identification Task from discussions with Prof. A. Carpentier (HEGP, Paris, France) and V. Falk (HerzZentrum, Leipzig, Germany). It turns out that the surgeon matches the relative positions of bifurcations and the shape of the vessel's groove with its mental representation of the coronary tree and uses some anatomical landmarks. This match is error prone even for highly skilled surgeons and in some cases the identification can not be performed or a wrong artery is grafted [2]. Thus, we propose to superimpose on the live endoscopic images, the preoperative model of the coronary tree as a visual aid to the surgeon to locate himself relatively to the heart and to guide him during the navigation to the target arteries. 
Image-guided surgery, addressed in various specialties such as laparoscopic surgery [3], breast [4] or neurosurgery [5], requires the registration of a preoperative model with the patient. The existing methods use some natural landmarks, artificial markers [5] and a locating device in the Operating Room (OR) 4]. The most advanced systems, mainly in neurosurgery, correct the intrasurgical deformations with intraoperative imaging and a deformation model [6].

The visual guidance in robot assisted surgery is a promising but difficult and not well documented approach especially for CABG. The operating conditions deform the thoracic cage and displace the heart but the shift can not be easily assessed with intraoperative imaging. The wet aspect of the tissues and the direct lighting from the endoscope, the fat hiding the arteries and the veins more visible, complexify the extraction of the arteries from the images. Moreover, as the Target Identification Task is performed before the arrest of the heart or its local stabilization, we have to deal with the beating heart. The local motion of a previously stabilized heart can be tracked in video images [7] but the reproducibility of a preoperative beating heart model should be more deeply analyzed as a sick heart may suffer from arrhythmia.

We overcome all these difficulties with an original approach to overlay a coronary tree model with a precision necessary to distinguish two neighboring arteries at one instant of the heart cycle: the diastole. An external registration provides the initial overlay and helps the surgeon to locate himself. Then (s)he defines some Virtual Pins on the landmarks observed on the heart surface while moving the endoscope. The measures are taken into account to correct in real-time the initial overlay and automatically identify the previously pointed landmarks through a multi-model estimation method. Guided by the overlay, the surgeon adds some new Virtual Pins to reach the precision necessary to a safe target localization. This guidance system is applied in the cardiac context but the algorithm can easily be extended to other surgical specialties by changing the nature of the landmarks and the preoperative model.

We describe the overall method, present the results of in vivo experiments before drawing trends for the future of our system.

\section{Material and Methods}

\subsection{Patient Imaging and Modeling}

The preoperative model of the patient is built from two acquisition modalities through a single interface. We have developed and integrated a method [8] to build a 3D model of the coronary tree from the angiograms used for cardiovascular diseases diagnostic. The surgeon models the essential arteries of the intervention: the occluded target arteries and the neighboring branches leading to potential intraoperative mistakes (Fig. 2(a). A set of labeled B-splines curves describes their 3D skeleton corresponding to the diastole (Fig. 2(c) on which the surgeon defines the site of the graft. The heart surface and the proximal part of the arteries is, so far, manually segmented and automatically reconstructed (Fig. 2(b) from an injected CT-scan used in CABG to assess the vessel wall 
quality and intramural position [9]. Radio-opaque markers sticked to the patient thorax for the transfer in OR [10] are segmented. The fusion of the two modalities in a single frame $\mathcal{F}_{\text {pre }}$ is currently manually performed by aligning the proximal part of the coronary tree segmented in the CT-scan. A volumetric rendering view of CT-scan eases the process by underscoring the arteries (Fig. $2(\mathrm{c})$ and $2(\mathrm{~d})$.

\subsection{Overlay Initialization}

In this initialization stage, we make the assumption of the rigidity of the set of markers sticked on the patient thorax with the target organ inside the patient body. The shift of the heart due to the operating conditions - left lung collapse and $\mathrm{CO}_{2}$ insufflation - will be corrected by intraoperative measurements. The procedure used for the transfer in the OR of the preoperative planning [10] is applied: the markers are pointed with the robot tool tip to compute [1] the transform between $\mathcal{F}_{\text {pre }}$ and the operative frame $\mathcal{F}_{\text {op }}$ (Fig. [1). The covariance matrix $\boldsymbol{\Lambda}_{\text {pre-op }}$ of its $[\mathbf{r}, \mathbf{t}]$ representation is derived [12].

Reliable position data are provided by the joints values of an actuated endoscope without any tracking system. We compute the optical and mechanical parameters of the stereoscopic endoscope and quantify the precision of the measured displacement with a planar calibration pattern [13. The RMS error in pixels between the real and virtual corners of the grid - for different positions of the endoscope corresponding to the exploration of the surgical volume - evaluates the precision of the mapping $\mathcal{F}_{\text {op }}$ to $\mathcal{F}_{\text {cam }^{\mathbf{j}}}$, frames of the endoscopic images.

Finally, the covariance matrix of $\mathbf{q}_{\mathbf{0}}=\left[\mathbf{r}_{\mathbf{0}}, \mathbf{t}_{\mathbf{0}}\right]$, parameter of the rigid transform applied to the coronary tree, is evaluated as the sum of $\boldsymbol{\Lambda}_{\mathbf{p r e}-\mathbf{o p}}$ and $\boldsymbol{\Lambda}_{\mathbf{d i s p l}}$ characterizing the intraoperative expected shift of the heart.
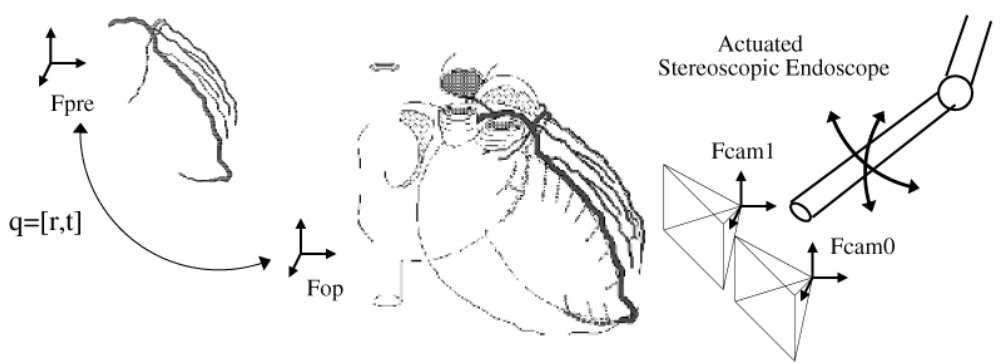

Fig. 1. The frames of the transfer in OR.

\subsection{Interactive Registration and Guidance}

Surgical Protocol: During the Target Identification Task, we ask the surgeon - guided by the initial overlay - to put, in any order, some Virtual Pins on the landmarks discovered while exploring the heart surface. The Virtual Pins are $2 \mathrm{D}$ or $3 \mathrm{D}$ beacons on the observed organ's surface, defined on the live or frozen endoscopic images. In the context of CABG, two types of Virtual Pins are defined: the junction of two arteries or a sliding point on an artery. In case of obvious landmarks potentially recognized by the surgeon, an option allow to 
enter hypotheses on the identity of the landmark. These measures correct in realtime the overlay and increase the precision of the guidance. The overall process takes a few minutes before the graft.

Measures Entry: A Virtual Pin is recorded as a 2D measure $\mathbf{p}_{\mathbf{i}}$, its covariance matrix $\boldsymbol{\Lambda}_{\mathbf{i}}$, the list $\mathbf{L}_{\mathbf{i}}$ of associated labels, its nature and the projection matrix $\mathbf{P}_{\mathbf{i}}^{\mathbf{j}}$ depending on the current endoscope position. If no hypothesis on the landmark identity was formulated, all the possible labels are considered. A corresponding point is searched in the second image. If the matching succeeds, this additional measure is added and the Virtual Pin is a 3D one. The uncertainty of the measure has different sources: $(1)$ the $\mathcal{F}_{\text {op }}$ to $\mathcal{F}_{\text {cam }^{j}}$ mapping, (2) the spatial inaccuracy of the pointing, (3) the possible displacement of the landmark due to a time-lag between the pointing and the heart's diastole, the non reproducibility of the heart motion or the breath (4) eventually the uncertainty due to the stereo-matching deduced from the correlation ratio curve [14].

Overlay Correction: Given a set $\mathbf{p}_{\mathbf{i}}$ of data labeled by $\mathbf{l}_{\mathbf{i}} \in \mathbf{L}_{\mathbf{i}}$ with related information matrix $\mathbf{Q}_{\mathbf{i}}$, inverse of the covariance, we estimate the parameter $\mathbf{q}=[\mathbf{r}, \mathbf{t}]$ of the rigid transform $\mathbf{D}(\mathbf{q})$, "close" to the a priori $\mathbf{q}_{\mathbf{0}}$ and the corrected measures $\tilde{\mathbf{p}}_{\mathbf{i}}$ "close" to the approximated ones verifying the equations binding measures and parameter. We use the quadratic distances $\left\|\mathbf{x}-\mathbf{x}_{\mathbf{0}}\right\|_{\mathbf{Q}}^{2}=\left(\mathbf{x}-\mathbf{x}_{\mathbf{0}}\right)^{\mathbf{T}} \mathbf{Q}\left(\mathbf{x}-\mathbf{x}_{\mathbf{0}}\right)$ defined by the information matrices [15] to write the estimation as an optimization problem:

$$
\min _{\left(\tilde{\mathbf{q}}, \tilde{\mathbf{p}_{1}}, \ldots, \tilde{\mathbf{p}_{i}}, \ldots, \tilde{\mathbf{p}_{\mathbf{n}}}\right)} \mathcal{L}^{2}=\frac{1}{2}\left\|\tilde{\mathbf{q}}-\mathbf{q}_{0}\right\|_{\mathbf{Q}_{0}}^{2}+\sum_{\mathbf{i}=1}^{\mathrm{n}} \frac{1}{2}\left\|\tilde{\mathbf{p}_{\mathbf{i}}}-\mathbf{p}_{\mathbf{i}}\right\|_{\mathbf{Q}_{\mathbf{i}}}^{2}
$$

given the measurement equations:

$$
\forall \mathbf{i} \in[\mathbf{1}, \mathbf{n}], \mathbf{c}_{\mathbf{i}}\left(\tilde{\mathbf{q}}, \tilde{\mathbf{p}_{\mathbf{i}}}\right)=\tilde{\mathbf{p}_{\mathbf{i}}}-\mathbf{P}_{\mathbf{i}}^{\mathbf{j}} \mathbf{D}(\tilde{\mathbf{q}}) \mathbf{M}_{\mathbf{i}}=\mathbf{0}
$$

In eq. (2), the $3 \mathrm{D}$ point $\mathbf{M}_{\mathbf{i}}$ is the junction $\mathbf{l}_{\mathbf{i}}$ of the model or a $3 \mathrm{D}$ point $\mathbf{M}_{\mathbf{i}}\left(\mathbf{t}_{\mathbf{i}}\right)$ sliding along the B-spline curve of the artery $\mathbf{l}_{\mathbf{i}}$. In this case, the additional parameter $\mathbf{t}_{\mathbf{i}}$ is simultaneously optimized. The criterion minimization is performed in a few iterations by linearizing the equations around the estimate. The formalism is flexible: if the a priori information $\left(\mathbf{q}_{\mathbf{0}}, \mathbf{Q}_{\mathbf{0}}\right)$ is available, a result is displayed even with no intraoperative measurements. Then, the overlay is corrected after the first pointed landmark and while new measures are proposed by the surgeon. On the other hand, if no a priori information is available, we write $\mathbf{Q}_{\mathbf{0}}=\mathbf{0}$ in eq.(1) but we need a minimal set of measures to estimate $\mathbf{q}$.

Managing Hypothesis with a Multi Model Approach: We define a model as a set of labeled measures and its corresponding estimate. The models are stored in a stack ordered by the normalized cost $\frac{\mathcal{L}^{2}}{\text { dof }}$. When a new measure is added, the models corresponding to the list $\mathbf{L}_{\mathbf{i}}$ of possible labels are generated and optimized. We prune the models tree by deleting the unrelevant ones. 
Thresholding unrelevant models is a statistical ill-posed problem and we have obtained better results by simply considering the relative relevance of the models and keeping the $n$-best models after the integration of each new measure. The "best" estimated overlay is displayed but if the surgeon is not satisfied, (s)he selects another model in the stack . The corresponding labels identify the landmarks on the operating field view (Fig. 3(b)].

Quantifying the Precision of the Overlay: To avoid a misleading overlay and a target mismatch, we define a precision indicator which depends on the geometry of the coronary artery tree and is relative to the point of view: the risk of mistake increases when the approach is low-angled and the neighboring arteries are closer. From eq.(1) and (2), the precision of the estimate $\tilde{\mathbf{q}}$ is derived, then, given a threshold $\alpha$, the ellipsoid of uncertainty around the current projection of a $3 \mathrm{D}$ target point. The overlay precision indicator is defined as the ratio between the size of this ellipsoid and the distance in the image from the target to the nearest artery.

\section{Experiments and Results}

The da Vinci ${ }^{\mathrm{TM}}$ surgical system was used for experiments with Prof. V. Falk at HerzZentrum, Leipzig, Germany. The overall approach is integrated in the single architecture STARS [1]. The two video channels of the endoscope are digitized simultaneously to the state of the robot. The live endoscopic images are processed and augmented on a separated monitor but a 3D augmentation inside the stereoscopic display of the master console is currently demonstrated.

The experiments were conducted on a sheep. The figure 2(a) shows the coronary tree model obtained from two mono-plane angiograms [8]. Although it is not especially error prone since the diagonal branches are not developed, it provides a good validation case. The heart surface and the radio-opaque markers were modeled from an injected CT-scan as well as the proximal part of the arteries (Fig. 2(b) to guide the fusion of the two modalities under the control of the volumetric rendering of the CT-scan data (Fig. 2(c) and 2(d). The $30^{\circ}$ actuated stereoscopic endoscope was calibrated [13] and the accuracy of the displacements inside the surgical volume was evaluated to 4 pixels. For registration, 10 markers were pointed on the sheep's shaved thorax with the robot tool tip. We obtained an RMS error of $1.1 \mathrm{~cm}$ which is higher than the precision of the robot and the markers segmentation. It points out the deformation of the set of markers sticked on the skin.

Due to the anatomy of the animal, the endoscope accessed to the heart in the unusual direction of the interventricular groove. The coronary tree was superimposed on the endoscopic images. The initial misalignment was quite high, combining different sources of error: the fusion of the two modalities, the robot, the external rigid registration and the operating conditions. The operating field was then explored to look for landmarks. The figure 3(a) is a snapshot of the initial overlay (left view of the endoscope). The two arteries on the lower-left 
corner are in background and should be hidden but this feature is not so far integrated. An obvious artery groove is visible. The figure 3(b) shows the correction of the overlay after the definition of two Virtual Pins (3D and 2D) on this groove and one junction (3D). The freezing of the image makes the pointing easier but we could observe the non exact periodicity of the heart beating (modeled by the measures uncertainty). Then the endoscope was moved to see the left side of the heart (Fig. 3(c) and a new artery could be observed (the overlay is simultaneously updated with the joint values of the endoscope). This artery was pointed and the overlay was corrected (Fig. $3(\mathrm{~d})$. The identification of the landmarks is visible on figure 3(b) but the multi-model approach could not significantly distinguish the two closed junctions at the image center.

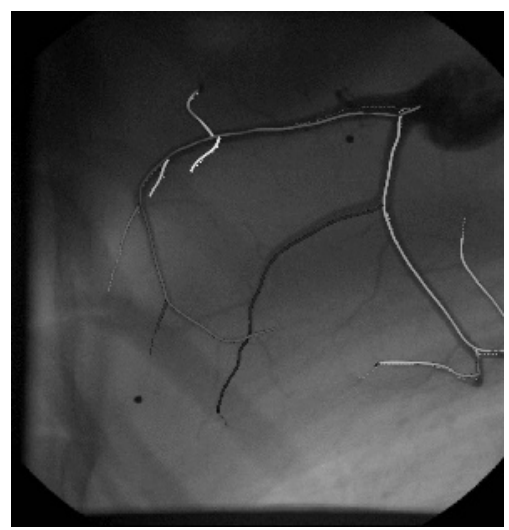

(a) Reprojection of the coronary tree $3 \mathrm{D}$ model on an angiogram.

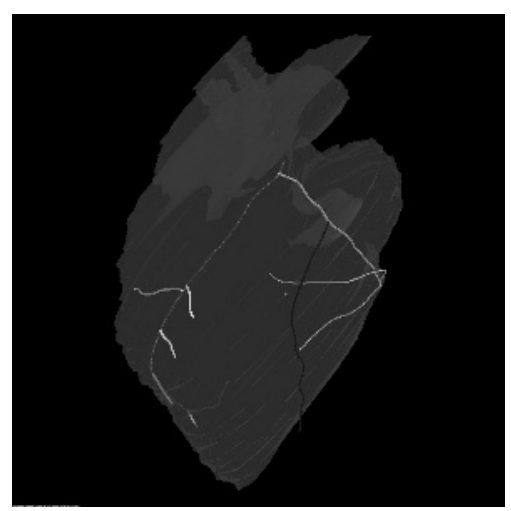

(c) Fusion of the heart surface model and the coronary tree from the angiography.

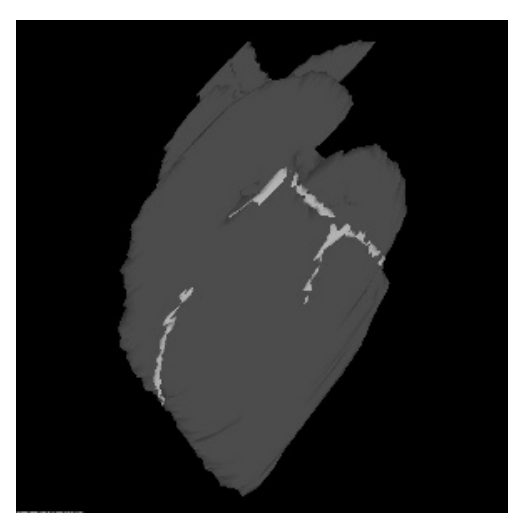

(b) Surface model of the heart and proximal part of the arteries from the injected CT scan.

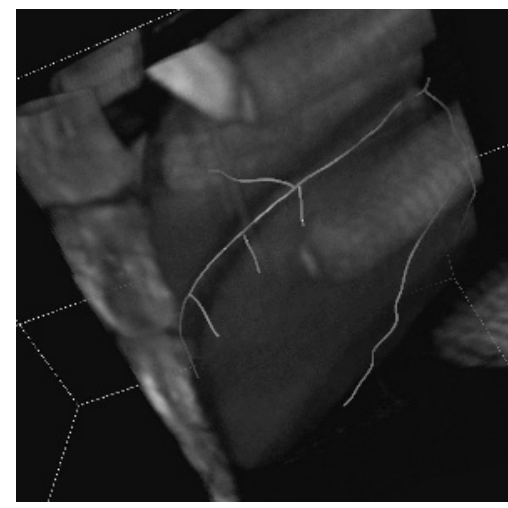

(d) Volumetric rendering of the injected CT scan and coronary tree from the angiography.

Fig. 2. Patient imaging and modeling. 


\section{Discussion and Future Trends}

We described an original visual guidance system in the especially difficult context of robot assisted CABG. It is based on the initial overlay of the coronary tree and the pointing by the surgeon of landmarks observed in the operating field during motions of the endoscope. The overlay is corrected in real-time and the landmarks are automatically identified through a multi-model estimation method until the precision necessary to the localization of targets is reached. Preliminary tests were conducted on a dog [10] and a newer in vivo experiment was presented here. The main arteries were identified in a few minutes and in a few clicks as confirmed by a thoracotomy performed a posteriori.

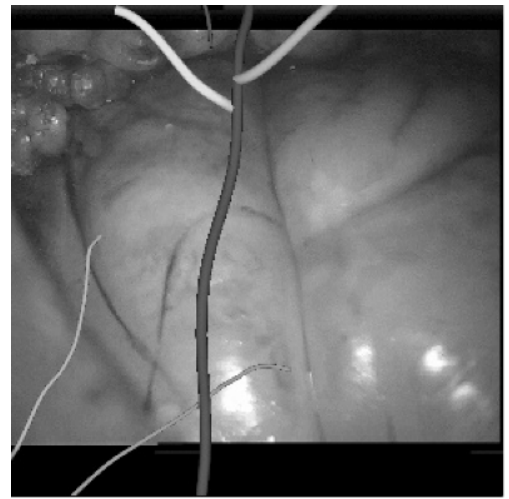

(a) Initial overlay of the coronary tree (left view of the endoscope).

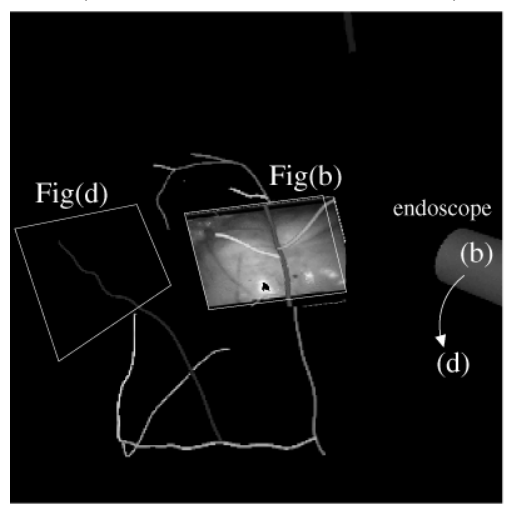

(c) Displacement of the endoscope (the overlay is updated during the displacement).

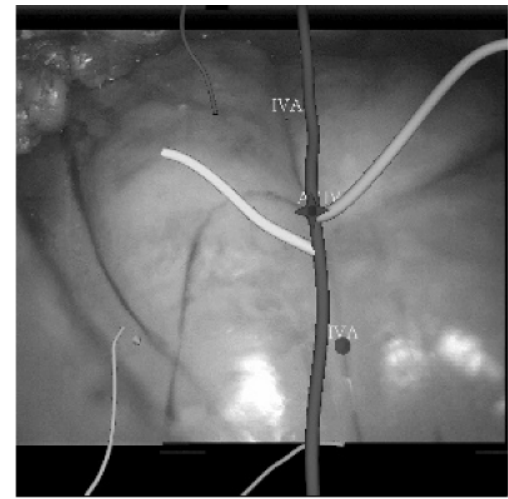

(b) Overlay after pointing a junction (3D) and the artery (3D and 2D).

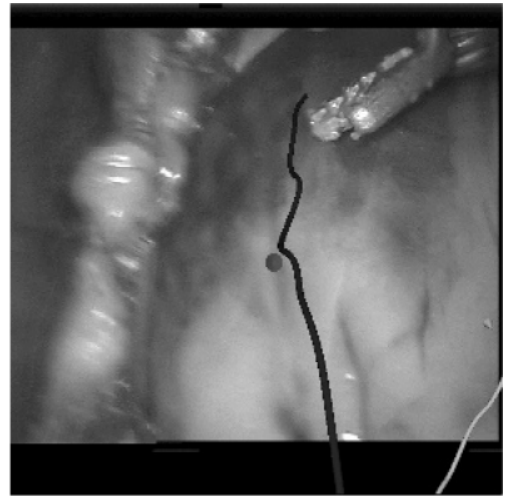

(d) Guided by the overlay, pointing (3D) of this new artery to correct the superimposition.

Fig. 3. Interactive guidance (see text for details).

This trial was the first of several additionnal experiments which are currently starting. The external registration can be improved by taking into account the 
non-isotropic uncertainty of the measures with the robot tool tip. Measuring and modeling the shift of the heart will lead to a better initial overlay and to a lighter registration method in the OR. Since a pointed landmark may be a vein or a junction not preoperatively modeled, a smarter hypothesis management technique including the outliers is currently tested in the interactive registration stage. Further work will also address the correction of possible heart deformations by extending the formalism used to estimate the rigid transform. Finally, the general ergonomics of the interface (e.g. masking the background arteries) is studied for its integration in the surgeon tool.

Acknowledgments. O. Faugeras is gratefully acknowledged for some fruitful ideas during this work. We also thank C. Coirier from Intuitive Surgical France for his technical help, and Prof. A. Carpentier from the Hôpital Européen Georges Pompidou, Paris, France, and the cardiac surgery team of Prof. F. Mohr at HerzZentrum, Leipzig, Germany, for their validation of the true clinical added value of this work.

\section{References}

1. Adhami, L., Coste-Manière, È.: A versatile system for computer integrated miniinvasive robotic surgery. In: Proc. of MICCAI. Volume 2488 of LNCS., Springer (2002) 272-281

2. Falk, V., Diegeler, A., Walther, T., Banusch, J., Brucerius, J., Raumans, J., Autschbach, R., Mohr, F.W.: Total endoscopic computer enhanced coronary artery bypass grafting. European Journal of Cardiothoracic Surgery 17 (2000) 38-45

3. Fuchs, H., Livingston, M., Raskar, R., Colucci, D., Keller, K., State, A., Crawford, J., Rademacher, P., Drake, S., A.Meyer: Augmented reality visualization for laparoscopic surgery. In: Proc. of MICCAI. Volume 1496 of LNCS., Springer (1998) 934-943

4. Sato, Y., Nakamoto, M., Tamaki, Y., Sasama, T., Sakita, I., Nakajima, Y., Monden, M., Tamura, S.: Image guidance of breast cancer surgery using $3 \mathrm{~d}$ ultrasound images and augmented reality visualization. IEEE Transactions on Medical Imaging 17 (1998) 681-693

5. Grimson, E., Leventon, M., Ettinger, G., Chabrerie, A., Ozlen, F., Nakajima, S., Atsumi, H., Kikinis, R., Black, P.: Clinical experience with a high precision imageguided neurosurgery system. LNCS 1496 (1998) 63-73

6. Audette, M., Siddiqi, K., Peters, T.: Level-set surface segmentation and fast cortical range image tracking for computing intra-surgical deformations. In: Proc. of MICCAI. Volume 1679 of LNCS., Springer (1999) 788-797

7. Gröger, M., Ortmaier, T., Sepp, W., Hirzinger, G.: Tracking local motion on the beating heart. In: Proc. of SPIE Medical Imaging Conference. Volume 4681. (2002) 233-241

8. Mourgues, F., Devernay, F., Malandain, G., Coste-Manière, E.: 3d+t modeling of coronary artery tree from standard non simultaneous angiograms. In: Proc. of MICCAI. Volume 2208 of LNCS., Springer (2001) 1320-1322

9. Dogan, S., Wimmer-Greinecker, G., Tayfun, A., Dzemali, O., Herzog, C., Vogl, T.J., Moritz, A.: Multidetector CT scan facilitates complex totally endoscopic coronary artery bypass grafting (TECAB). The Heart Surgery Forum 5 (2001) $239-250$ 
10. Coste-Manière, غ̀., Adhami, L., Mourgues, F., Bantiche, O., etal.: Optimal planning of robotically assisted heart surgery: Transfer precision in the operating room. In Siciliano, B., Dario, P., eds.: Springer Tracts In Advanced Robotics, Experimental Robotics VIII. Volume 5., Springer (2002) 424-434

11. Faugeras, O., Hebert, M.: The representation, recognition, and locating of 3-D shapes from range data. International Journal of Robotics Research $\mathbf{5}$ (1986) 27-52

12. Pennec, X., Thirion, J.P.: A framework for uncertainty and validation of 3D registration methods based on points and frames. The International Journal of Computer Vision 25 (1997) 203-229

13. Mourgues, F., Coste-Manière, È.: Flexible calibration of actuated stereoscopic endoscope for overlay in robot assisted surgery. In: Proc. of MICCAI. Volume 2488 of LNCS., Springer (2002) 25-34

14. Yi, S., Haralick, R.M., Shapiro, L.G.: Error propagation in machine vision. Machine Vision and Applications 7 (1994) 93-114

15. Vieville, T., Lingrand, D., Gaspard, F.: Implementing a multi-model estimation method. The International Journal of Computer Vision 44 (2001) 41-64 